\title{
Splanchnic metabolism of propylene glycol infused into the jugular vein of steers under washed rumen conditions*
}

\author{
B.M.L. Raun ${ }^{1}$, N.B. Kristensen ${ }^{1,3}$ and D.L. Harmon ${ }^{2}$ \\ ${ }^{1}$ Department of Animal Nutrition and Physiology, \\ Danish Institute of Agricultural Sciences \\ Box 50, DK-8830 Tjele, Denmark \\ ${ }^{2}$ Department of Animal Sciences, University of Kentucky \\ Lexington, KY 40546, USA
}

\begin{abstract}
The splanchnic metabolism and blood to rumen flux of propylene glycol (PG) infused into the jugular vein of steers, implanted with permanent indwelling catheters in major splanchnic blood vessels as well as a rumen cannula, was investigated under washed rumen conditions. Up to $95 \%$ of the infused PG was taken up in splanchnic tissues. The hepatic uptake of PG accounted for up to $70 \%$ of the PG infused though the hepatic extraction ratio was only $8 \%$. Up to $9 \%$ of the infused PG was transferred to the buffer incubated in the washed rumen. Increased hepatic balance of L-lactate could account half of the hepatic PG uptake. In conclusion the liver is the quantitatively most important organ for PG uptake from the blood, however, up to about one third of blood PG could be transferred back to the lumen of the gastrointestinal tract. L-lactate is a quantitatively important product of PG metabolism in the ruminant liver.
\end{abstract}

KEY WORDS: propylene glycol, splanchnic metabolism, steer

\section{INTRODUCTION}

In a previous study it was observed that PG accumulated in the blood of cows when absorbed from the washed rumen and no response to PG was observed for the whole body irreversible flux of glucose (Kristensen et al., 2002). The aim of the present study was to investigate the splanchnic metabolism of PG infused into the jugular vein of steers and test the hypothesis that blood PG is partly transferred to the lumen of the gastrointestinal tract and therefore have a higher availability for microbial metabolism than apparent from the relative fermentation and absorption rates of PG in the rumen.

\footnotetext{
* Supported by the Danish Agriculture and Veterinary Research Council and the University of Kentucky Agriculture Experimental Station

${ }^{3}$ Corresponding author: e-mail: nbk@agrsci.dk
} 


\section{MATERIAL AND METHODS}

Three Holstein steers fitted with permanent indwelling catheters in the mesenteric artery, mesenteric vein, hepatic portal vein, hepatic vein, and in the right ruminal vein as well as a ruminal cannula (\#2C rumen cannula, Bar Diamond Inc., Parma, ID) were sampled $70 \pm 21$ days after surgery, with an average body weight of $302 \pm 20 \mathrm{~kg}$. One steer was sampled per sampling day. Continuous infusion of para-aminohippuric acid (pAH, $17.3 \pm 0.7 \mathrm{mmol} / \mathrm{h}$ ) into the mesenteric vein and propylene glycol $(\mathrm{PG}, 119 \pm 2 \mathrm{mmol} / \mathrm{h})$ into the jugular vein was initiated. The rumen was emptied, washed, and incubation of VFA buffers, as well as continuous intraruminal VFA infusion to maintain VFA absorption, was initiated as previously described (Kristensen and Harmon, 2004). The steers were subjected to 3 buffer incubations each of $125 \mathrm{~min}$. The rumen was emptied and washed between each of the buffer incubations. Blood samples were simultaneously drawn from the arterial, portal, and hepatic catheters 60,90 and $120 \mathrm{~min}$ after initiation of each buffer incubation. Plasma concentrations of PG were determined by the method described by Needham et al. (1982) with the following modifications: plasma (450 $\mu \mathrm{L}$ ) was added $100 \mu \mathrm{L} 5 \mathrm{mM} \mathrm{1,2} \mathrm{butanediol} \mathrm{as} \mathrm{internal} \mathrm{standard} \mathrm{and} \mathrm{deproteinized}$ with $4 \mathrm{~mL}$ of acetonitrile. The supernatant was dried under a stream of nitrogen at $70^{\circ} \mathrm{C}$. One milliliter of $1 \% \mathrm{p}$-bromophenylboric acid in ethyl acetate was added and incubated for $30 \mathrm{~min}$. The sample was dried and resuspended in $100 \mu \mathrm{L}$ of cyclohexane. Samples were analysed by gas chromatography (Varian 3400, Palo Alto, USA), using a HP Ultra-1 column ( $25 \mathrm{~m}, 0.3 \mathrm{~mm} \mathrm{ID}, 0.17 \mu \mathrm{m}$ film) at a head pressure of $8 \mathrm{psi}$ and an initial temperature of $60^{\circ} \mathrm{C}$ increased at $10^{\circ} \mathrm{C} / \mathrm{min}$ to $200^{\circ} \mathrm{C}$. Plasma concentrations of propionate, L-lactate, D-3-hydroxybutyrate, glucose, L-glutamate, and L-glutamine were determined as described previously (Kristensen and Harmon, 2004). Net portal fluxes and net hepatic fluxes were calculated as previously described (Kristensen and Harmon, 2004). Means within animal and buffer incubation were analysed by the repeated measures ANOVA of the GLM procedure of SAS (SAS Inst., Inc., Cary, NC). Net portal flux and net hepatic fluxes of lactate, glucose, 3-hydroxybutyrate, propionate, glutamate, and glutamine observed in the present study were compared to observations on the same three animals under identical experimental conditions except for the PG infusion by a paired $t$-test.

\section{RESULTS AND DISCUSSION}

The arterial concentration of $\mathrm{PG}$ increased $(\mathrm{P}=0.001)$ with time of infusion reaching $1.79 \mathrm{mmol} / \mathrm{L}$ blood in the third period (Table 1 ). In agreement with a lower infusion rate of PG, relative to the body weight in the present study, the arterial concentration of PG remained below the concentrations found in a 
previous study with dairy cows (Kristensen et al., 2002). The net portal flux of PG was negative, indicating uptake of arterial PG across the PDV (portal drained viscera), however, the net portal flux was not affected by period $(\mathrm{P}=0.75)$. The net hepatic flux of PG was negative and about three times larger than the net portal flux. The PG uptake in the liver increased with period $(\mathrm{P}=0.01)$, the largest numerical increase was from period 1 to 2 . The hepatic extraction ratio was $8 \%$, but despite of a relatively low hepatic extraction ratio the liver removed more than $70 \%$ of the PG infused during period 2 and 3 . The splanchnic tissues removed $95 \%$ of the infused PG during period 3 . The generally larger differences observed between period 1 and 2 compared with the differences between 2 and 3 could indicate that infusion and utilization of PG was approaching a steady and that that PG during the first hours of infusion was accumulating in body fluids.

Table 1. Arterial concentration, net portal flux, net hepatic flux, and hepatic extraction ratio of propylene glycol (PG) as well as the proportion of propylene glycol infused into the jugular vein, taken up in splanchnic tissues in steers under washed rumen conditions

\begin{tabular}{lccccc}
\hline \multirow{2}{*}{ Item } & \multicolumn{3}{c}{ Period } & \multirow{2}{*}{ SEM } & P \\
\cline { 2 - 4 } & 1 & 2 & 3 & & \\
\hline Arterial concentration, mmol/L & 0.89 & 1.45 & 1.79 & 0.17 & 0.001 \\
Net portal flux, mmol/h & -18 & -27 & -28 & 41 & 0.60 \\
Net hepatic flux, mmol/h & -56 & -84 & -85 & 2 & 0.11 \\
$\begin{array}{l}\text { Hepatic extraction ratio } \\
\begin{array}{l}\text { Proportion of infused PG taken } \\
\text { up in splanchnic tissues }\end{array}\end{array}$ & 0.08 & 0.08 & 0.07 & 0.005 & 0.66 \\
\hline
\end{tabular}

The ruminal concentration of $\mathrm{PG}$ at the end of ruminal incubations, the ruminal accumulation rate of $P G$ and the fraction of the hourly infused PG recovered in the ruminal buffer increased with period $(\mathrm{P}<0.05$; Table 2$)$. In average $30 \%$ of the PG taken up in the PDV was recovered in the ruminal buffer. To what extent the PDV tissues metabolized PG or all PDV uptake was simply transfer to the lumen of the gastrointestinal tract is not known. However, due to the fact that $20 \%$ of the infused PG was taken up across the PDV and $30 \%$ of that was found in the rumen, PG seems to have a higher availability to microbes in the gastrointestinal tract than inferred from it relative high absorption rate (Clapperton and Czerkawski, 1972). 
Table 2. Ruminal propylene glycol (PG) concentration, ruminal accumulation rate of PG, and fraction of the hourly infusion rate of PG recovered in the ruminal buffer of steers infused with PG in the jugular vein under washed rumen conditions. All values are calculated from samples obtained after $120 \mathrm{~min}$ incubation of ruminal buffers in 3 subsequent periods

\begin{tabular}{lcccccc}
\hline \multirow{2}{*}{ Item } & \multicolumn{3}{c}{ Period } & \multirow{2}{*}{ SEM } & $\mathrm{P}$ \\
\cline { 2 - 4 } & 1 & 2 & 3 & & 0.01 & 0.002 \\
\hline Ruminal concentration, mmol/1 & 0.41 & 0.74 & 0.96 & & 0.4 & 0.005 \\
Ruminal accumulation, mmol/h & 4.3 & 8.0 & 10.2 & & 0.003 & 0.01 \\
$\begin{array}{l}\text { Fraction of infused PG recovered } \\
\quad \text { in ruminal buffer }\end{array}$ & 0.04 & 0.07 & 0.09 & & \\
\hline
\end{tabular}

There was no effect of PG infusion on the net portal fluxes of propionate, lactate, glucose, 3-hydroxybutyrate, glutamate, and glutamine or on the net hepatic fluxes of propionate, glucose, 3-hydroxybutyrate, glutamate, and glutamine $(\mathrm{P}>0.10$; data not shown). However, the net hepatic flux of lactate changed $(\mathrm{P}<0.01)$ from minus 19 to plus $21 \pm 11 \mathrm{mmol} / \mathrm{h}$. The increased lactate balance across the liver accounted for $56 \pm 12 \%$ of the PG uptake in the liver. This could indicate that only the (S)-1,2-propanediol is metabolized into L-(S)-lactate.

\section{CONCLUSIONS}

Up to $95 \%$ of intravenously infused propylene glycol (PG) was taken up by the splanchnic tissues. The hepatic extraction ratio was relatively low (8\%) for PG, but the liver was the quantitatively most important organ for PG metabolism and released L-lactate to peripheral tissues, equivalent to half of the PG uptake.

\section{REFERENCES}

Clapperton J.L., Czerkawski J.W., 1972. Metabolism of propane-1:2-diol infused into the rumen of sheep. Brit. J. Nutr. 27, 553-560

Kristensen N.B., Danfær A., Røjen B.A., Raun B.-M.L., Weisbjerg M.R., Hvelplund T., 2002. Metabolism of propionate and 1,2-propanediol absorbed from the washed reticulorumen of lactating cows. J. Anim. Sci. 80, 2168-2175

Kristensen N.B., Harmon D.L., 2004. Splanchnic metabolism of VFA absorbed from the washed reticulorumen of steers. J. Anim. Sci. (in press)

Needham L.L., Hill R.H. Jr., Orti D.L., Felver M.E., Liddle J.A., 1982. Electron-capture, capillary column gas chromatographic determination of low-molecular-weight diols in serum. J. Chromatogr. 233, 9-17 